\title{
PREDICTED X-RAY FLUXES OF STELLAR CORONAS
}

\author{
C. DE LOORE and C. DE JAGER \\ Astronomical Institute Brussels, Belgium, and \\ Astronomical Institute Utrecht, The Netherlands
}

\begin{abstract}
Models of convection zones and corresponding mechanical energy fluxes were computed for 90 stellar photospheres with effective temperatures ranging from $2500 \mathrm{~K}$ to $41600 \mathrm{~K}$ and acceleration of gravity values between 1 and $10^{5}$. The most intense X-ray fluxes may be expected from stars with $T \approx 7200 \mathrm{~K}$ and $\operatorname{lag} g \approx 4$. Detectable X-ray fluxes could be expected from Procyon, $\alpha$ Cen and $\beta$ Cas.
\end{abstract}

\section{Computed Convective Models of Stellar Photospheres}

Models of convective layers in stars were computed by one of us (De Loore, 1970), for 90 models of stellar photospheres for effective temperatures ranging from 2500 to $41600 \mathrm{~K}$ and values for the acceleration of gravity, $g$, between 1 and $10^{5}$. The computations were based on Böhm-Vitense's (1958) mixing length theory. Mechanical fluxes were computed on the basis of Lighthill's considerations.

The computations show that there are two temperature ranges for which the largest values of the mechanical fluxes may be expected. These are first around $7200 \mathrm{~K}$ and secondly also around $3700 \mathrm{~K}$. The variation of the mechanical flux with effective temperature and $g$-values is shown in Figures 1 and 2.

\section{Expected Stellar Coronas}

With the hypothesis that the mechanical energy flux is responsible for the heating of the corona, coronal models were constructed for the sun and for a 10 stars with effective temperatures between $5000 \mathrm{~K}$ and $8320 \mathrm{~K}$ for $\log g$-values of 4 or 5 .

For main sequence stars the largest fluxes are generated in F-stars; stars with $T_{\text {eff }}=7130 \mathrm{~K}$ and $\log g=4$ possess also the hottest and almost dense coronas, with a computed temperature of $3.7 \times 10^{6} \mathrm{~K}$ and $\log N_{\mathrm{e}}=10.5$.

The solar corona computed in this way, on the basis of a photospheric mechanical flux of $0.14 \times 10^{8} \mathrm{erg} \mathrm{cm}^{-2} \mathrm{sec}^{-1}$, has a temperature of $1.3 \times 10^{6} \mathrm{~K}$ and $\log N_{\mathrm{e}}=9.8$. This density is apparently too high, but even when including in the computations all theoretical refinements proposed in the last few years by various authors it does not appear possible to obtain a solar coronal model with a smaller density.

\section{Expected Stellar X-Ray fluxes}

In estimating the expected stellar X-ray fluxes we make our computations in a differential way, by comparing them with the solar values. In that way possibly occurring small imperfections in the computations are hopefully greatly eliminated. 


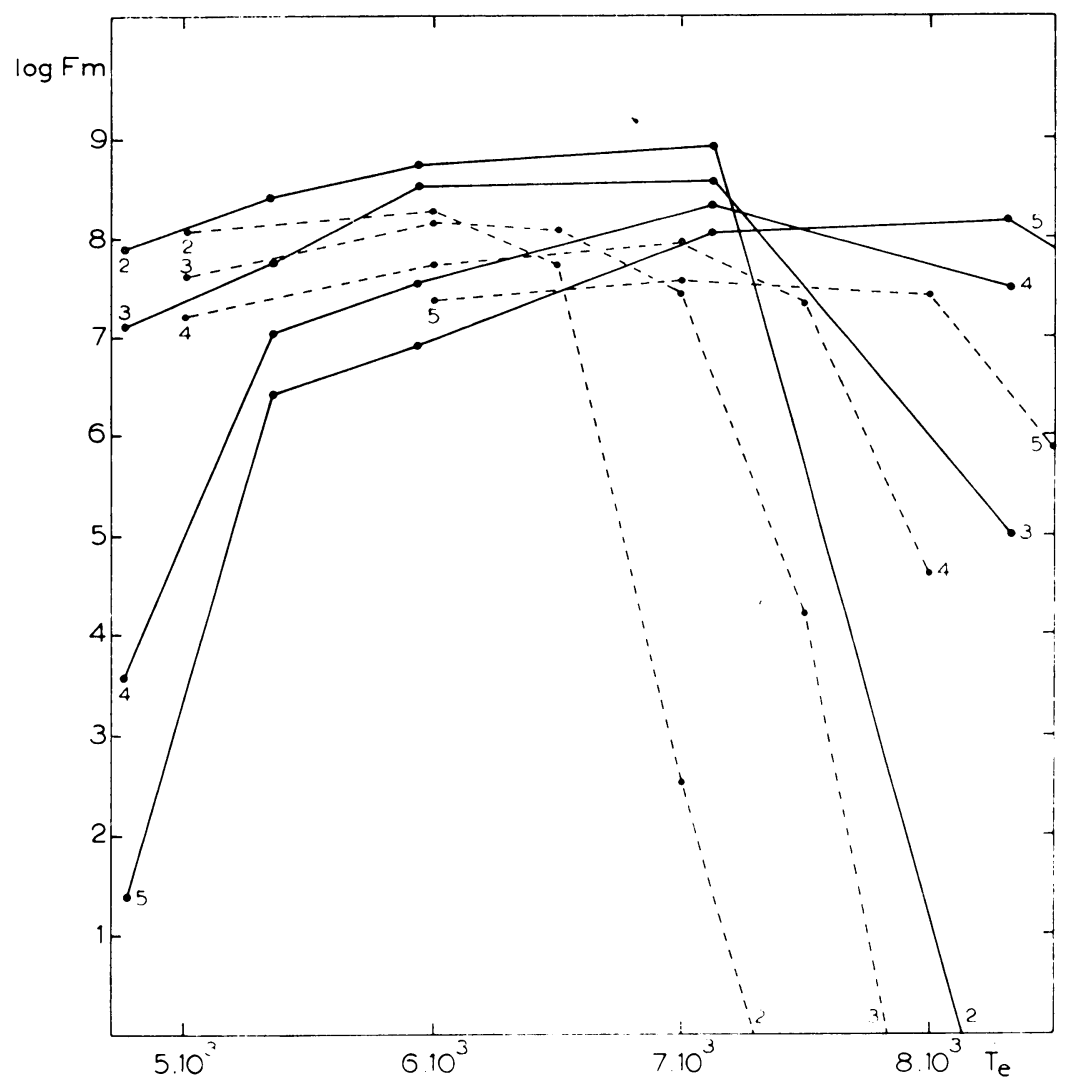

Fig. 1. Computed mechanical fluxes for stars. The curves are labelled with their $\log g$-values. Dashed are recent computational results of Nariai (1969).

The ratio between the expected X-ray flux of a star and that of the sun is given by:

$$
\frac{F_{\mathrm{X}}(\mathrm{star})}{F_{\mathrm{X}}(\mathrm{sun})}=\left(\begin{array}{l}
R_{\text {star }} \\
R_{\text {sun }}
\end{array}\right)^{2}\left(\begin{array}{l}
N_{\mathrm{e} \mathrm{star}} \\
\hdashline N_{\mathrm{e} \text { sun }}
\end{array}\right)^{2}\left(\begin{array}{c}
H_{\text {star }} \\
H_{\text {sun }}
\end{array}\right)\left(\begin{array}{l}
d_{\text {sun }} \\
d_{\text {star }}
\end{array}\right)^{2},
$$

where $R$ and $d$ are the star's radius and distance; $N_{\mathrm{e}}$ is the electron density at the basis of the stellar corona; $H$ is the coronal scale height.

From Allen (1962), we extracted the spectral types, the absolute magnitudes and the distances of a number of favourable stars. From the statistical relations, given in Allen, between the spectral type, the absolute magnitude, the radii, and the acceleration of gravity, we obtained values of the stellar radii and their $g$-values.

From the effective temperatures and $g$-values the temperatures and electron densities of the stellar coronas could be derived on the basis of the computations described in Section 2 of this paper.

Since the coronas are expected to be optically thin, the predicted X-ray flux may be proportional to the square of the electron densities. The precise value of the coronal temperature is less important for the predicted X-ray flux. 


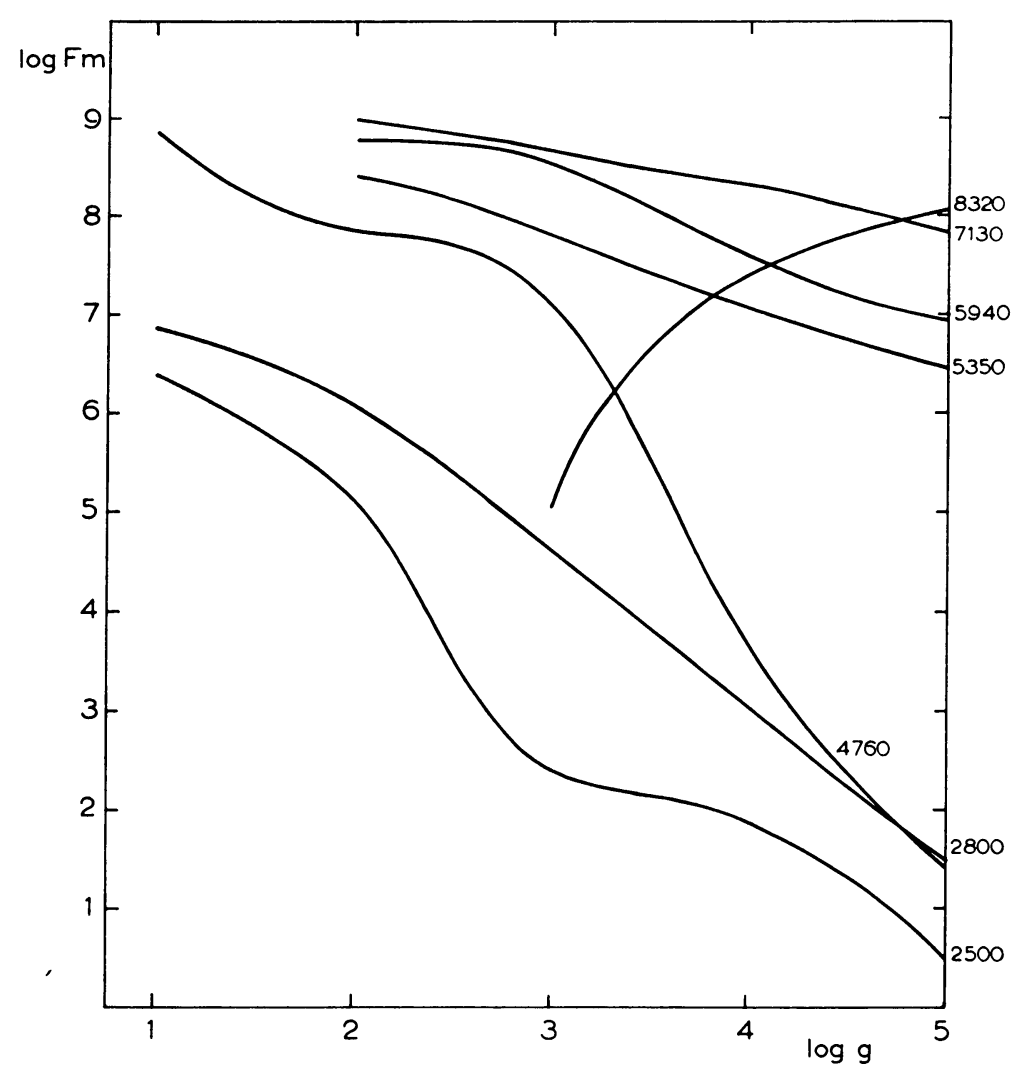

Fig. 2. The same results as those of Figure 1, now plotted against $\log g$, with the effective temperature as a parameter.

In comparing the predicted X-ray fluxes with those of the sun we used Friedman's (1959) observations, yielding a total solar X-ray flux during sunspot minimum of $0.13 \mathrm{erg} \mathrm{cm}^{-2} \mathrm{sec}^{-1}$ and during the maximum he found values ranging up to $1 \mathrm{erg} \mathrm{cm}^{-2} \mathrm{sec}^{-1}$. These are values integrated over the whole $\mathrm{X}$-range. The corresponding numbers of photons are between 0.2 and $1.5 \times 10^{9}$ photons $\mathrm{cm}^{-2} \mathrm{sec}^{-1}$ at earth's distance.

The results are found in Table I.

TABLE I

\begin{tabular}{|c|c|c|c|c|c|c|c|c|}
\hline \multirow{2}{*}{$\begin{array}{l}\text { Star } \\
\text { Procyon }\end{array}$} & \multirow{2}{*}{$\begin{array}{l}\text { Spectrum } \\
\text { F5 }\end{array}$} & \multirow{2}{*}{$\begin{array}{l}T_{\text {eff }} \\
6500\end{array}$} & \multirow{2}{*}{$\begin{array}{l}\log g \\
4.3\end{array}$} & \multirow{2}{*}{$\begin{array}{l}\log \left(R / R_{\text {sun }}\right) \\
0.2\end{array}$} & \multirow{2}{*}{$\begin{array}{l}\begin{array}{l}\text { Corona } \\
\log \left(N / N_{\text {sun }}\right)\end{array} \\
+0.86\end{array}$} & \multirow{2}{*}{$\frac{\begin{array}{l}\text { logdistance } \\
\text { (parsec) }\end{array}}{0.55}$} & \multicolumn{2}{|c|}{$\begin{array}{l}\text { Expected integral } \\
\text { photon fluxes } \\
\text { (cm-2 } \mathrm{sec}^{-1} \text { earth) }\end{array}$} \\
\hline & & & & & & & 0.016 & 0.12 \\
\hline & G2 & 5800 & 4.4 & 0.1 & 0 & 0.11 & 0.008 & 0. \\
\hline Cas & F2 & 6900 & 4.5 & 0.1 & +1.20 & 1.15 & 0.0007 & 0.005 \\
\hline Sun & G2 & 5800 & 4.4 & 0 & 0 & -5.3 & $0.2 \times 10^{9}$ & $1.5 \times 10^{9}$ \\
\hline
\end{tabular}


In interpreting these expected integral photon fluxes it should be remembered that these values are the integral ones. With a given detector, which mostly only observes in a restricted wavelength band, the flux may easily be reduced by a factor of 100 . Nevertheless, even when taking this into account, it does not look impossible that within a few years observations of the X-ray fluxes of Procyon, $\alpha$ Cen and perhaps also of $\beta$ Cas, could be obtained.

\section{References}

Allen, C. W.: 1962, Astrophysical Quantities, Athlone Press, London, 2nd ed. Böhm-Vitense, E.: 1958, Z. Astrophys. 46, 108.

De Loore, C.: 1970, Astrophys. Space Sci. 6, 60.

Friedman, H.: 1959, Proc. Inst. Res. Eng. 47, 272.

Nariai, K.: 1969, Astrophys. Space Sci. 3, 150, 160. 